\title{
Inhibition of the mTOR/S6K signal is necessary to enhance fluorouracil-induced apoptosis in gastric cancer cells with $H E R 2$ amplification
}

\author{
HIDEO TOMIOKA $^{1}$, TORU MUKOHARA ${ }^{1,2}$, YU KATAOKA ${ }^{1}$, ROUDY CHIMINCH EKYALONGO ${ }^{1}$, \\ YOHEI FUNAKOSHI ${ }^{1}$, YASUO IMAI ${ }^{3}$, NAOMI KIYOTA ${ }^{1}$, YUTAKA FUJIWARA ${ }^{1,4}$ and HIRONOBU MINAMI ${ }^{1,2}$ \\ ${ }^{1}$ Department of Internal Medicine, Division of Medical Oncology/Hematology, Kobe University Graduate \\ School of Medicine; ${ }^{2}$ Kobe University Hospital Cancer Center, Kobe 650-0017; ${ }^{3}$ Department of Pathology, \\ Dokkyo Medical University Koshigaya Hospital, Koshigaya, Saitama 343-8555, Japan
}

Received January 30, 2012; Accepted April 3, 2012

DOI: $10.3892 /$ ijo.2012.1485

\begin{abstract}
The purpose of this study was to explore the effect of trastuzumab in enhancing the activity of chemotherapeutic agents and the molecular basis of this effect. Two gastric cancer cell types with HER2 amplification, one sensitive (NCI-N87) and one insensitive (MKN-7) to trastuzumab, were tested for the effects of trastuzumab on cell growth and cell signaling using MTS assay and western blotting, respectively. Interaction between trastuzumab and chemotherapeutic agents (fluorouracil, doxorubicin, cisplatin and paclitaxel) was evaluated by the combination index (CI). Fluorouracil-induced apoptosis was evaluated using western blot for poly (ADP-ribose) polymerase (PARP). Trastuzumab decreased phosphorylation of S6K, showed synergistic effect with fluorouracil or doxorubicin, and increased fluorouracil-induced apoptosis in NCI-N87 cells, but not in MKN-7 cells. While the mTOR inhibitor everolimus enhanced fluorouracil-induced apoptosis in both HER2amplified cell lines, this was not the case in the gastric cancer cell lines without HER2 amplification. Consistently, while the EGFR/HER2 inhibitor CL-387,785 inhibited cell growth of MKN-7, this growth inhibition did not accompany decrease in phosphorylation of S6K, and the compound did not enhance fluorouracil-induced apoptosis. In summary, inhibition of the mTOR/S6K signal may be a key molecular event in enhancing fluorouracil-induced apoptosis specifically in gastric cancer cells with HER2 amplification. mTOR inhibitors may therefore
\end{abstract}

Correspondence to: Dr Toru Mukohara, Department of Medical Oncology and Hematology, Kobe University Hospital, 7-5-2 Kusunoki-cho, Chuo-ku, Kobe 650-0017, Japan

E-mail: mukohara@med.kobe-u.ac.jp

Present address: ${ }^{4}$ Department of Internal Medicine and Thoracic Oncology, National Cancer Center Hospital, Tsukiji 5-1-1, Chuo-ku, Tokyo 104-0045, Japan

Key words: gastric cancer, trastuzumab, HER2, everolimus, fluorouracil be attractive alternative drugs in gastric cancers with $H E R 2$ amplification regardless of their sensitivity to trastuzumab.

\section{Introduction}

Gastric cancer is the fourth-most common cancer and the second leading cause of cancer death worldwide $(1,2)$. When diagnosed in its early stages, a surgical approach with a partial or total gastric resection are often successful (3). However, when diagnosed with primarily unresectable disease or recurrence after surgery (4), the disease is no longer curable despite systemic chemotherapy with cytotoxic chemotherapeutic agents, including fluorouracil, cisplatin, doxorubicin and paclitaxel, either alone or in combination. Response rates to these chemotherapies are generally less than $50 \%$ and median survival time from diagnosis is less than one year (5). New treatment strategies are therefore urgently required.

Molecularly targeted agents, particularly those targeting receptor tyrosine kinases (RTKs), have been successfully used in clinical settings since the late 1990s. The most promising of these have generally been those targeting RTKs with genetic changes causing aberrant activation. These include trastuzumab, a monoclonal antibody against extracellular domain of human epidermal growth factor receptor 2 (HER2), in HER2-overexpressing breast cancer (6); and erlotinib and gefitinib, which inhibit epidermal growth factor receptor (EGFR) with somatic mutations in non-small cell lung cancer $(7,8)$. In gastric cancer, gene amplification in HER2, MET, and fibroblast growth factor receptor 2 (FGFR2) have been reported in $20 \%$, $20 \%$, and $40 \%$ of tumor samples, respectively. While HER2 amplification is predominantly found in the well-differentiated intestinal subtype (9-14), MET and FGFR2 amplification occur more frequently in the undifferentiated diffuse subtype (15). The potential of these RTKs as therapeutic targets in gastric cancer was clinically demonstrated by the success of the ToGA (Trastuzumab for Gastric Cancer) study, at least in part (16). This open-label, international, phase III randomized controlled trial compared cisplatin plus fluorouracil or capecitabine (reference arm) versus the same regimens combined with trastuzumab in HER2-overexpressing advanced gastric cancer. Results showed 
that the trastuzumab-containing arm was superior to chemotherapy alone with regard to response rate, progression-free survival, and overall survival (16).

Although the ToGA trial shed light on the strategy of targeting gene-amplified RTK in gastric cancer, several questions remained to be answered, including whether trastuzumab enhances the effect of chemotherapeutic agents even in trastuzumab-resistant HER2-amplified gastric cancer cells, and the control of trastuzumab-resistant HER2-amplified cells.

Here, we compared the effects of trastuzumab in two HER2-amplified gastric cancer cell lines in vitro, one of which has been reported to be sensitive to anti-HER2 monoclonal antibody (NCI-N87) and the second to be insensitive (MKN-7).

\section{Materials and methods}

Cell culture. NCI-N87 cells were purchased from American Type Culture Collection (ATCC, Manassas, VA, USA). NUGC-4, MKN-7, KATO-III, MKN-45, MKN-1, and MKN-74 cells were purchased from RIKEN BioResource Center (Tsukuba, Japan). Both NCI-N87 and MKN-7 cells have been reported to have amplification of the HER2 gene $(17,18)$. NCI-N87 cells were reported to be sensitive to trastuzumab in vitro (19), while MKN-7 cells were shown to be resistant to monoclonal antibody 4D5, which has the same antigen-binding fragment (Fab) as trastuzumab (20).

All cell lines were maintained in RPMI-1640 (Cellgro) supplemented with $10 \%$ fetal bovine serum (FBS), $100 \mathrm{U} / \mathrm{ml}$ penicillin, $100 \mathrm{U} / \mathrm{ml}$ streptomycin, and $2 \mathrm{mM}$ glutamine. All cells were grown at $37^{\circ} \mathrm{C}$ in a humidified atmosphere with $5 \% \mathrm{CO}_{2}$ and were in the logarithmic growth phase upon initiation of the experiments. The cells were passaged for $\leq 3$ months before fresh cells were obtained from frozen early-passage stocks received from the indicated sources.

Drugs.Evelorimus (RAD001), an inhibitor of mTOR, was kindly provided by Novartis Pharma (Basel, Switzerland). Fluorouracil, cisplatin, doxorubicin and paclitaxel were purchased from Wako (Osaka, Japan). Trastuzumab was obtained from the Kobe University Hospital Pharmacy. CL-387,785, an inhibitor of EGFR/HER2, was purchased from Calbiochem (San Diego, CA, USA). Stock solutions were prepared in dimethyl sulfoxide (DMSO) and stored at $-20^{\circ} \mathrm{C}$. The drugs were diluted in fresh media before each experiment, with final DMSO concentrations less than $0.1 \%$.

Antibodies and western blotting. Cells were washed with icecold PBS and scraped immediately after the addition of lysis buffer [20 mM Tris ( $\mathrm{pH} 7.5$ ), $150 \mathrm{mM} \mathrm{NaCl}, 10 \%$ glycerol, $1 \%$ NP40, and 2 mM EDTA] containing protease and phosphatase inhibitors [100 mM NaF, $1 \mathrm{mM}$ phenylmethylsulfonyl fluoride (PMSF), $1 \mathrm{mM} \mathrm{Na} \mathrm{VO}_{4}, 2 \mu \mathrm{g} / \mathrm{ml}$ aprotinin, and $5 \mu \mathrm{g} / \mathrm{ml}$ leupeptin). Lysates were centrifuged at 14,000 relative centrifugal force for $10 \mathrm{~min}$. Supernatants were collected as protein extracts and then separated by electrophoresis on 7.6\% sodium dodecyl sulfate (SDS)-polyacrylamide gels, followed by transfer to nitrocellulose membranes (Millipore, Billerica, MA, USA) and detection by immunoblotting using the enhanced chemiluminescence system (New England
Nuclear Life Science Products, Boston, MA, USA). HER2/ ErbB2 (44E7), phospho-HER2/ErbB2 (Tyr1221/1222)(6B12), Akt, phospho-Akt (Ser473)(D9E), p70 S6 kinase, phospho-p70 S6 kinase (Thr389), and cleaved-PARP (Asp214)(D64E10) antibodies were purchased from Cell Signaling Technology (Beverly, MA, USA). Phospho-EGFR (Y1068), ERK1/2, phospho-ERK1/2 (pT185/pY187) antibodies were purchased from Invitrogen (Carlsbad, CA, USA), and $\beta$-actin antibody was purchased from Sigma-Aldrich (St. Louis, MO, USA). Immunoblot quantification was carried out by densitometry using ImageJ software (21).

Cell growth assay. Growth inhibition was assessed using the MTS assay (Promega, WI, USA), a colorimetric method for determining the number of viable cells based on the bioreduction of 3-(4,5-dimethylthiazol-2-yl)-5-(3-carboxymethoxyphenyl)2-(4-sulfophenyl)-2H-tetrazolium (MTS) to a soluble formazan product, which is detectable by spectrophotometry at a wavelength of $490 \mathrm{~nm}$. Cells were diluted in $160 \mu \mathrm{l} /$ well of maintenance cell culture media and plated in 96-well flatbottom plates (Corning, Lowell, MA, USA). After a 144-h growth period, the number of cells required to obtain an optical density (OD) within the linear range of the assay, 1.3-2.2, was determined for each cell line with pilot experiments. The number of cells per well used in the subsequent experiments was as follows: NCI-N87: 5,000; MKN-7: 3,000; KATO-III: 3,000; MKN-1: 2,000; MKN-45: 1,500; MKN-74: 2,000 and NUGC-4: 2,000. At $24 \mathrm{~h}$ after plating, cell culture media were replaced with $10 \%$ FBS containing media with and without drugs followed by incubation for an additional $120 \mathrm{~h}$. A total of 6 to 12 replicate wells were established for each experimental point and all experiments were performed at least in triplicate. Data are expressed as a percentage of growth relative to that of untreated control cells.

Combination index (CI). Interaction between trastuzumab and cytotoxic chemotherapeutic agents was evaluated by the median-effect method (CalcuSyn software; Biosoft) to determine the well-established CI (22), with which CI values $\leq 1$, and $>1$ indicate synergistic, additive, and antagonistic effects, respectively (23). The maximum concentration of trastuzumab was set at $100 \mu \mathrm{g} / \mathrm{ml}$, on the basis that this concentration is close to the maximum plasma concentration of trastuzumab observed clinically, and has been widely used in previous studies (24). Maximum concentrations of fluorouracil, doxorubicin, cisplatin, and paclitaxel was set at $100 \mu \mathrm{M}, 1 \mu \mathrm{M}, 10 \mu \mathrm{M}$, and $100 \mathrm{nM}$, respectively, based on their approximate $\mathrm{IC}_{90}$ values as determined by pilot experiments using the NCI-N87 cell line (data not shown). Serial two-fold dilution with a fixed concentration ratio of chemotherapeutic drug/trastuzumab was done, and total CI was represented as the average of CI at each experimental point.

\section{Results}

Effect of trastuzumab on cell growth in gastric cancer cell lines. We first screened seven gastric cancer cell lines for in vitro growth inhibition by trastuzumab (Fig. 1A). Results confirmed that trastuzumab was not effective in five non-HER2-amplified gastric cancer cell lines $\left(\mathrm{IC}_{50}>100 \mu \mathrm{g} / \mathrm{ml}\right)$. Of the two HER2- 
A

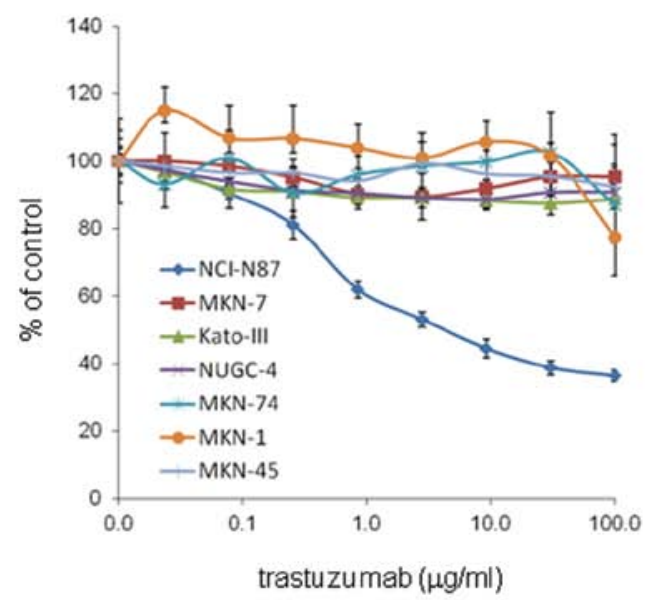

B

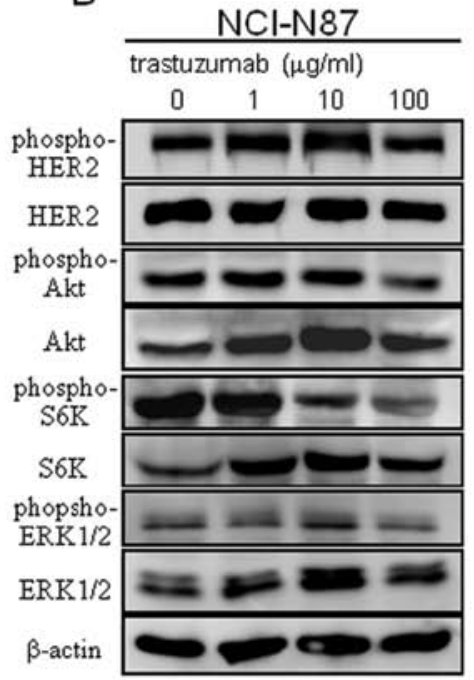

MKN-7

trastuzumab $(\mu \mathrm{g} / \mathrm{ml})$

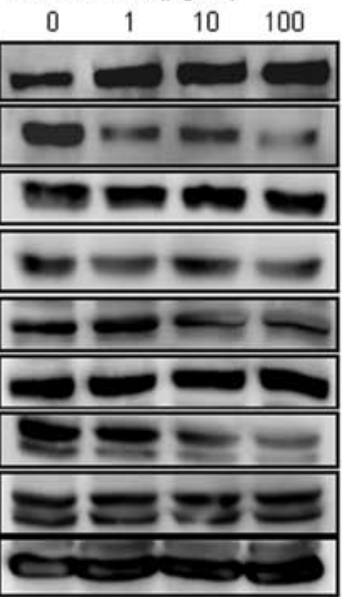

Figure 1. (A) Effect of trastuzumab on growth inhibition in seven gastric cancer cell lines in vitro. NCI-N87 and MKN-7 are HER2-amplified cell lines, and the others are HER2-non amplified cell lines. Gastric cancer cells were grown in $10 \%$ serum-containing media for 5 days in the presence of various concentrations of trastuzumab. The percentage of viable cells is shown relative to that of the untreated control and plotted on the $y$-axis, and trastuzumab concentration is plotted on the x-axis. Each data point represents the mean value and standard deviation of 6-12 replicate wells. (B) Expression of phosphorylated HER2 and representative downstream molecules in HER2-amplified gastric cancer cell lines with and without treatment with trastuzumab (100 $\mu \mathrm{g} / \mathrm{ml})$. Cells grown in $10 \%$ serum-containing media were lysed and immunoblotted for each protein. Blots were stripped and re-probed for $\beta$-actin as loading control.

amplified cell lines, only NCI-N87, and not MKN-7, was more sensitive than non-HER2-amplified cells, consistent with previous studies $(19,20)$.

Effect of trastuzumab on cell signaling in NCI-N87 and $M K N-7$ cell lines. To explore the underlying mechanism of the differential effect of trastuzumab in NCI-N87 and MKN-7, we examined phosphorylation of HER 2 and representative downstream signaling molecules in 10\% FBS-containing media with and without increasing the concentration of trastuzumab. The most remarkable difference in signaling outcome after trastuzumab treatment was a decrease in phosphorylation of S6K, which was observed only in NCI-N87, and not in MKN-7 (Fig. 1B).

Synergistic effect of trastuzumab and cytotoxic drugs in NCI-N87 cell line. We next investigated synergy between trastuzumab and the clinically relevant cytotoxic drugs fluorouracil, cisplatin, doxorubicin and paclitaxcel in HER2amplified NCI-N87 and MKN-7. In trastuzumab-sensitive NCI-N87, the combination of trastuzumab and fluorouracil or doxorubicin resulted in a CI value of 0.14 [95\% confidence interval (CI) -0.11-0.39] and 0.30 (95\% CI, -0.15-0.75), respectively, indicating significant synergy between the drugs (Fig. 2A and B). In trastuzumab-insensitive MKN-7, in contrast, trastuzumab and the individual chemotherapeutic drugs resulted in a $95 \%$ confidence interval for the CI value crossing 1 , which indicates no significant synergy between the drugs (Fig. 2C and D).

Fluorouracil-induced apoptosis in HER2-amplified gastric cancer cells. Because the combination of trastuzumab and fluorouracil showed the most promising synergy in the NCI-N87 cell line (Fig. 2A and B), we decided to explore the mechanism of the synergistic effect of the drugs in subsequent experiments.

To evaluate the level of apoptosis, we used western blot analysis for cleaved poly (ADP-ribose) polymerase (PARP), which is indicative for apoptosis. In the NCI-N87 cell line, while trastuzumab by itself produced a slight increase in cleaved PARP compared to control, the combination of trastuzumab and fluorouracil resulted in even higher level of cleaved PARP compared to either drug alone (Fig. 3A and $\mathrm{B}$ ). In MKN-7, in contrast, trastuzumab alone did not produce an increase in cleaved PARP compared to control, while the combination of trastuzumab and fluorouracil did not increase cleaved PARP expression compared to either drug alone (Fig. 3A and B). These results indicated that the synergy observed in NCI-N87 cells treated with trastuzumab and fluorouracil (Fig. 2A and B) was likely attributable to the enhancement of fluorouracil-induced apoptosis by trastuzumab. They also indicate that the synergistic effect of trastuzumab and fluorouracil may be limited to cell lines sensitive to trastuzumab itself.

Effect of everolimus in NCI-N87 and MKN-7 cell lines. Because a decrease in the phosphorylation of S6K on treatment with trastuzumab was observed only in NCI-N87, and not in MKN-7 (Fig. 1B), we hypothesized that inhibition of the phosphorylation of S6K may be an important molecular event for trastuzumab in its enhancement of fluorouracil-induced apoptosis and synergistic effect with the drug. Therefore, we next evaluated the effect of the mTOR inhibitor everolimus on cell signaling and fluorouracil-induced apoptosis in the two 
A
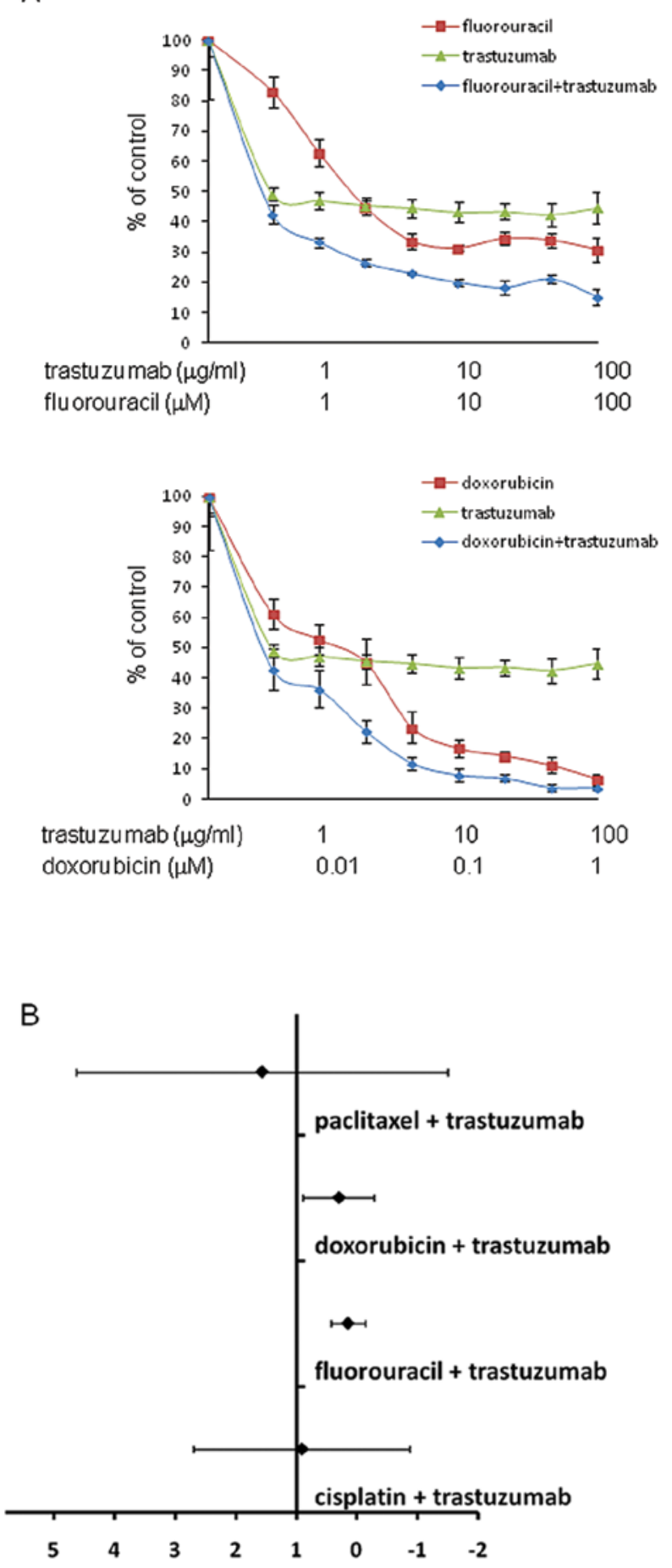

Figure 2. (A and C) Effect of trastuzumab, chemotherapeutic agents, and their combination on cell growth in NCI-N87 and MKN-7 cell lines in vitro. The percentage of viable cells is shown relative to that of the untreated control and plotted on the $y$-axis, and concentrations of each drug are plotted on the $\mathrm{x}$-axis. Each data point represents the mean value and standard deviation of 6-12 replicate wells.

HER2-amplified gastric cell lines. As seen in Fig. 4A, while everolimus alone inhibited phosphorylation of S6K, it instead decreased the expression of cleaved PARP as compared to control in both cell lines. When combined with fluorouracil, however, everolimus inhibited phosphorylation of S6K and appeared to enhance fluorouracil-induced apoptosis in a dose-
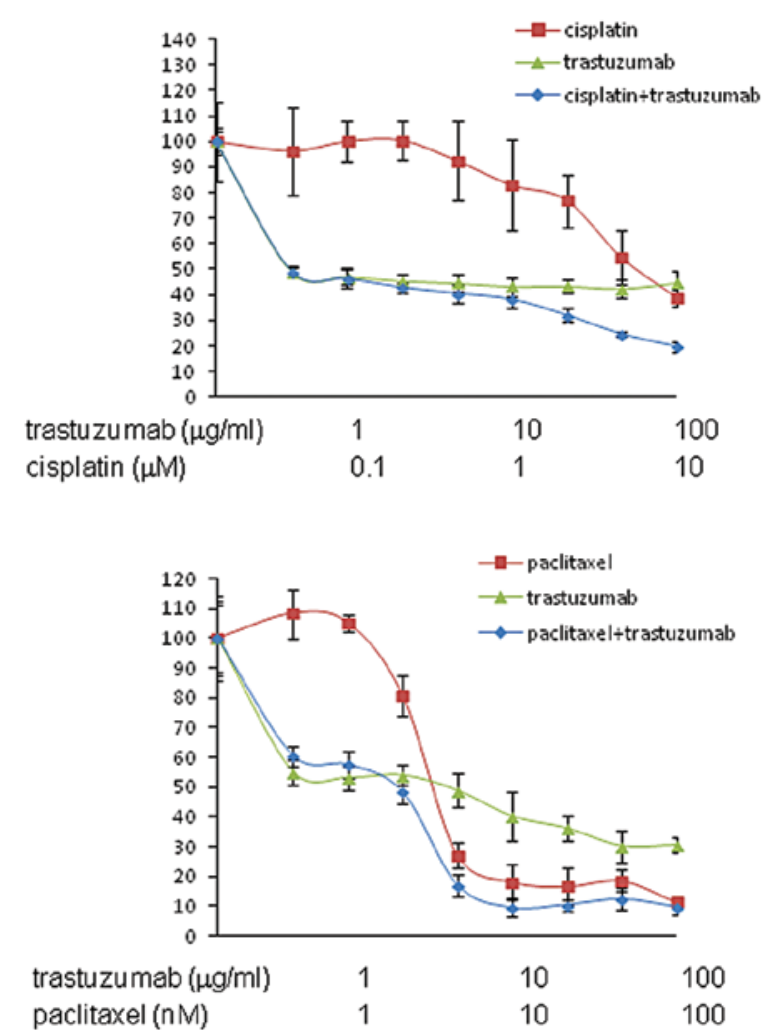

dependent manner in both cell lines (Fig. 4B). These results strengthen the notion that inhibition of the phosphorylation of S6K be a key molecular event in enhancing fluorouracilinduced apoptosis.

Effect of fluorouracil in combination with CL-387,785 in $M K N-7$. MKN-7 was previously shown to be resistant to anti-HER2 monoclonal antibody 4D5 because of alternative signaling from overexpressing EGFR (17). Therefore, we next evaluated the effect of CL-387,785, an EGFR/HER2 inhibitor, on cell growth, cell signaling and fluorouracil-induced apoptosis in MKN-7.

As seen in Fig. 5A, treatment of MKN-7 with CL-387,785 resulted in growth inhibition, albeit to a lesser extent than in NCI-N87. In the western blot analysis, treatment of CL-387,785 resulted in decrease in phosphorylation of ERK1/2 versus only a mild decrease in that of S6K (Fig. 5B). Consistent with this, the addition of CL-387,785 to fluorouracil did not increase the expression of cleaved PARP compared to fluorouracil alone (Fig. 5B). This finding again supports the notion that inhibition of the mTOR-S6K signal may be important in enhancing fluorouracil-induced apoptosis. Further, it suggests that growth inhibitory effect of anti-RTK agent alone does not guarantee its ability to enhance chemotherapy-induced apoptosis.

Synergistic effect of everolimus in combination with fluorouracil in the second gastric cancer cell line. To evaluate whether the mTOR-S6K signal in fluorouracil-induced apoptosis is universally important in all gastric cancers, we next tested other gastric cancer cell lines for fluorouracil-induced apoptosis. MKN-45, KATO-III, NUGC-4, MKN-1 and MKN-74, 

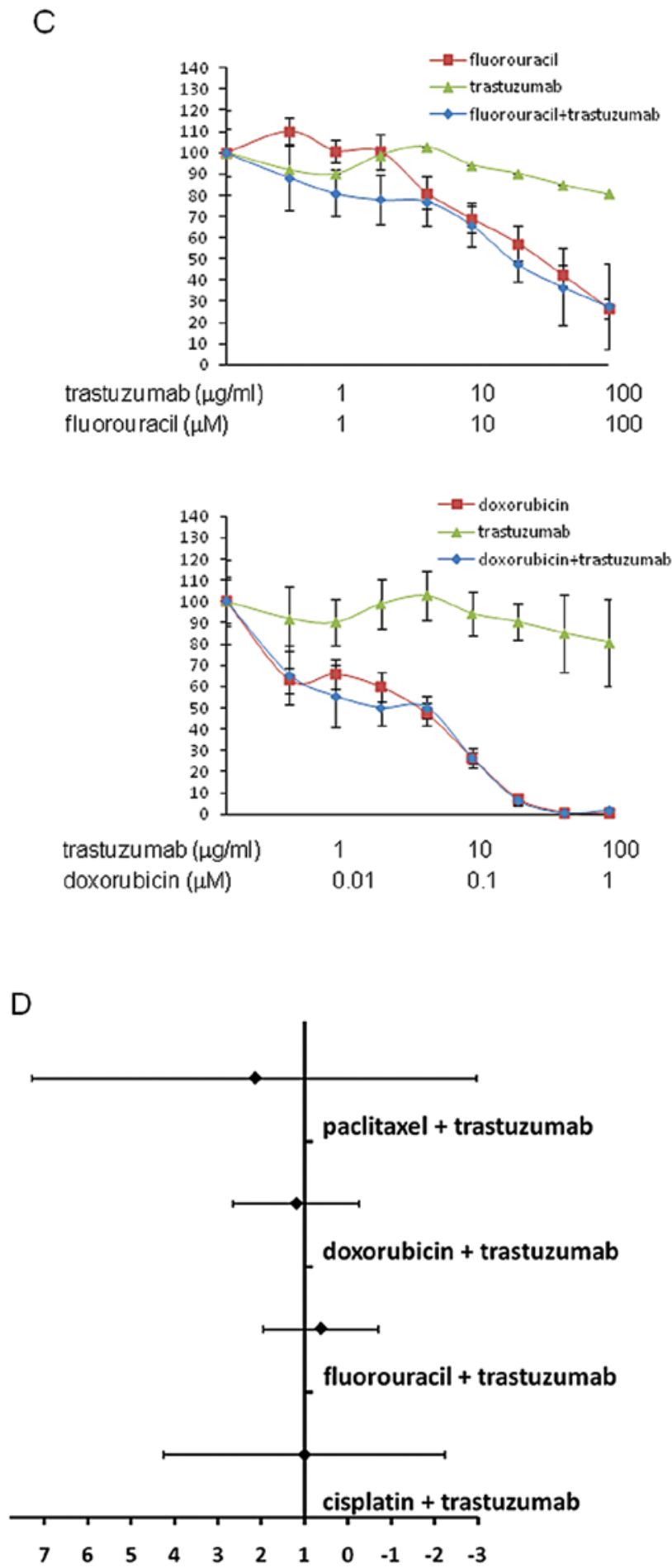

Figure 2. Continued. (B and D) Mean CI values for chemotherapeutic drugtrastuzumab combinations in NCI-N87 and MKN-7 cell lines. Error bars indicates $95 \%$ confidence intervals of the mean value.

which have been reported to harbor $M E T$-amplification (25), $F G F R 2$-amplification (25), EGFR-mutation (E746-A750 del), PIK3CA-mutation (E545K) (26), and B-RAF mutation (G466V), respectively (27), were treated with everolimus, fluorouracil, or their combination. Phosphorylation of S6K and cleavage of PARP were analyzed as shown in Fig. 6. Everolimus dramatically decreased the phosphorylation of S6K in all cell lines except MKN-74, while the combination
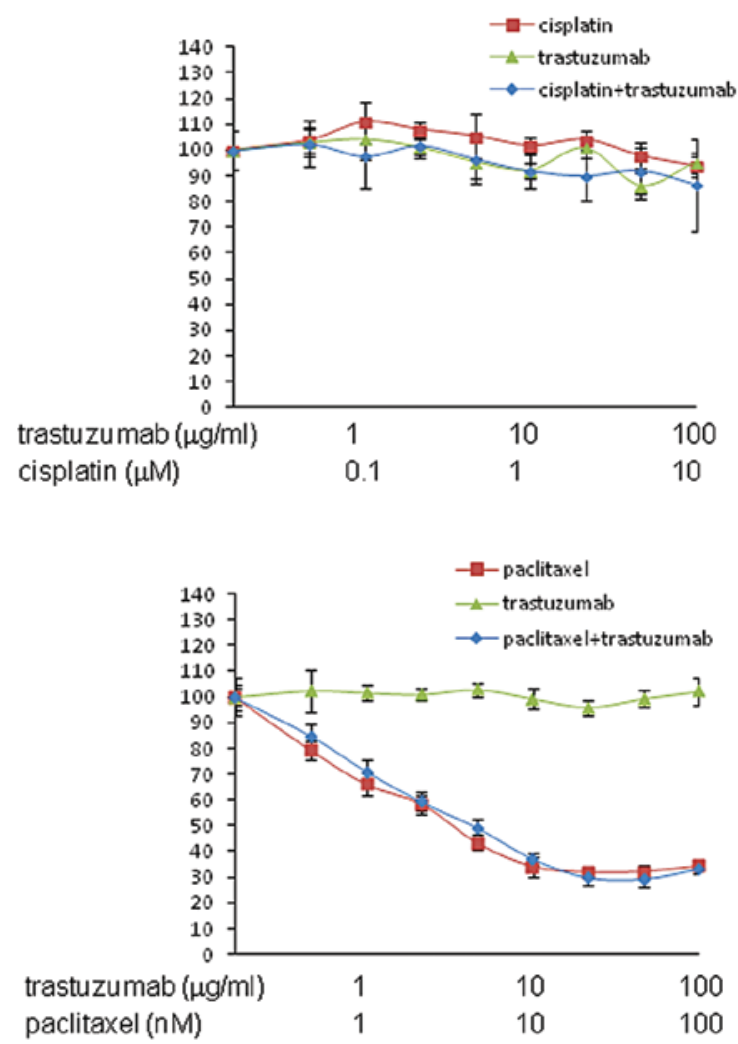

of fluorouracil and everolimus did not increase cleaved PARP expression compared to fluorouracil alone in any of the cell lines. This finding suggests that the mTOR-S6K signal in fluorouracil-induced apoptosis is not universal, but somewhat specific to HER2-amplified gastric cancer cell lines.

\section{Discussion}

In this study, we found that while trastuzumab administered together with fluorouracil or doxorubicin synergistically enhanced apoptosis in the trastuzumab-sensitive HER2amplified gastric cancer cell line NCI-N87, no such effect was seen in trastuzumab-insensitive HER2-amplified MKN-7 cells. We also found that inhibition of the phosphorylation of S6K appeared to be a key molecular event in the enhancement of fluorouracil-induced apoptosis, indicating that the mTOR inhibitor everolimus might be an attractive back-up drug for HER2-amplified gastric cancers.

Several previous studies evaluated the ability of trastuzumab to produce synergy with chemotherapeutic agents in HER2amplified gastric cancer cell lines. Kim et al (28) reported a synergistic effect between trastuzumab and cisplatin but only an additive effect between trastuzumab and fluorouracil or oxaliplatin using the SNU-216 cell line. Tanizaki et al (29) reported synergy between trastuzumab and fluorouracil in three HER2-amplified gastric cancer cell lines, NCI-N87, MKN-7, and SNU-216. These inconsistent results among the studies, including our present study, may be due to differences in experimental conditions. Moreover, none of the previous studies reported the drug concentrations used to test synergy between trastuzumab and chemotherapeutic agents. 
A

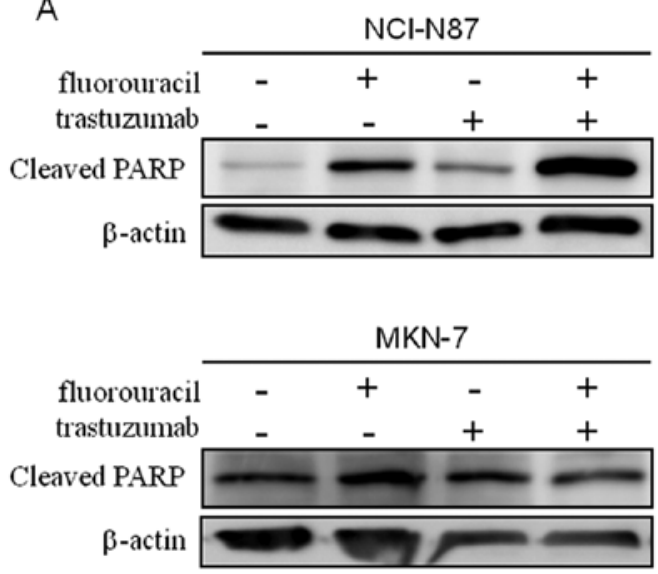

B

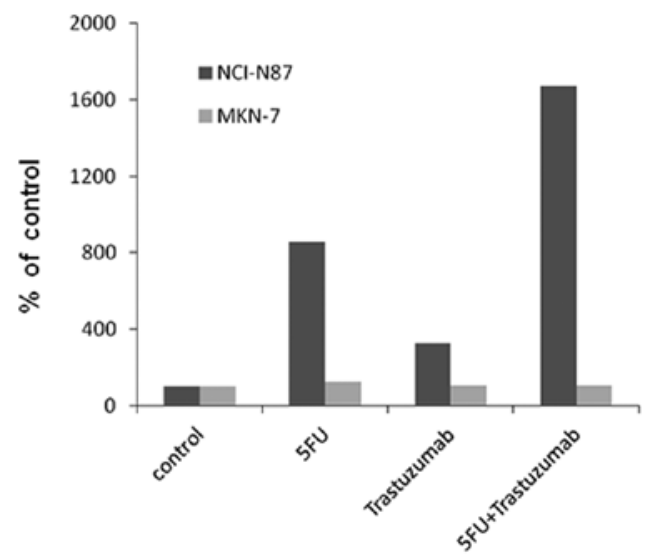

Figure 3. (A) Expression of cleaved PARP in HER2-amplified gastric cancer cell lines with and without treatment with fluorouracil (10 $\mu \mathrm{M})$ or trastuzumab $(100 \mu \mathrm{g} / \mathrm{ml})$. Cells grown in 10\% serum-containing media were lysed and immunoblotted for each protein. Blots were stripped and re-probed for $\beta$-actin as loading control. (B) The immunoblot was quantified by densitometry. Level of cleaved PARP was plotted on the y-axis as ratio to untreated control.

A

\begin{tabular}{|c|c|c|c|c|c|}
\hline \multirow[b]{2}{*}{ everolimus (nM) } & \multicolumn{5}{|c|}{ NCl-N87 } \\
\hline & 0 & 0.1 & 1 & 10 & 100 \\
\hline phospho-S6K & & & & & \\
\hline Cleaved PARP & 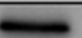 & ( & $\bar{\square}$ & 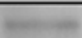 & \\
\hline$\beta$-actin & & & & & \\
\hline
\end{tabular}

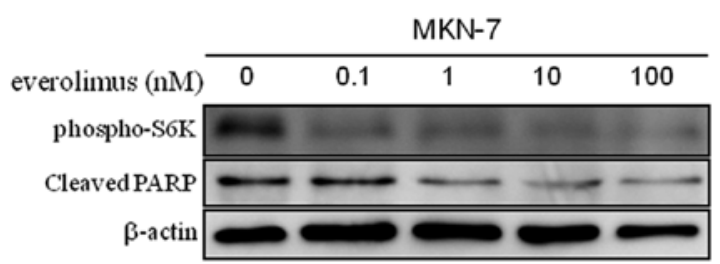

B

\begin{tabular}{|c|c|c|c|c|c|c|}
\hline \multirow[b]{2}{*}{ fluorouracil $(\mu \mathrm{M})$} & \multicolumn{6}{|c|}{$\mathrm{NCl}-\mathrm{N} 87$} \\
\hline & 0 & 10 & 10 & 10 & 10 & 10 \\
\hline everolimus (nM) & 0 & 0 & 0.1 & 1 & 10 & 100 \\
\hline \multicolumn{7}{|l|}{ phospho-S6K } \\
\hline \multicolumn{7}{|l|}{ CleavedPARP } \\
\hline$\beta$-actin & & & & & & \\
\hline
\end{tabular}

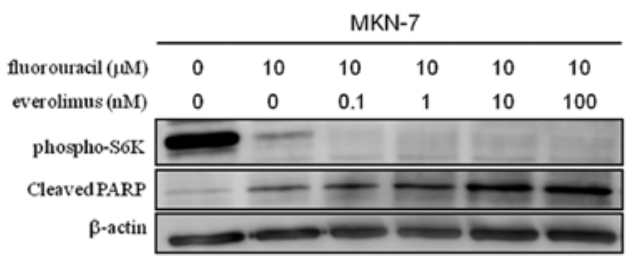

Figure 4. Expression of phosphorylated S6K and cleaved PARP in HER2-amplified gastric cancer cell lines with increasing concentrations of everolimus alone (A) or in combination with fluorouracil (B). Cells grown in 10\% serum-containing media were lysed and immunoblotted for each protein. Blots were stripped and re-probed for $\beta$-actin as loading control.

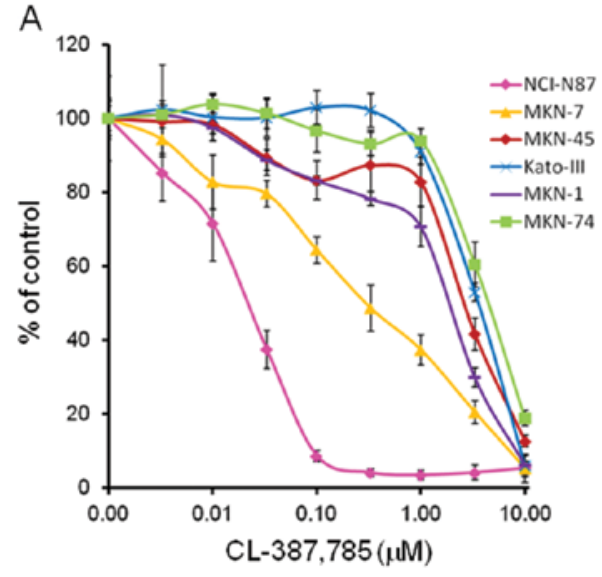

B

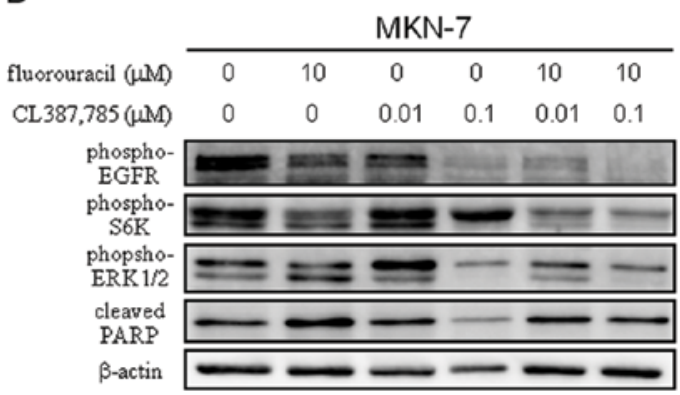

Figure 5. (A) Effect of CL-387,785 on growth inhibition in six gastric cancer cell lines in vitro. Cells were grown in $10 \%$ serum-containing media for 5 days in the presence of various concentrations of trastuzumab. The percentage of viable cells is shown relative to that of the untreated control and plotted on the $y$-axis, and concentrations of CL-387,785 are plotted on the x-axis. Each data point represents the mean value and standard deviation of 6-12 replicate wells. (B) Expression of phosphorylated EGFR and representative downstream molecules in $H E R 2$-amplified gastric cancer cell lines with increasing concentration of CL-387,785 or in combination with fluorouracil. Cells grown in 10\% serum-containing media were lysed and immunoblotted for each protein. Blots were stripped and re-probed for $\beta$-actin as loading control. 


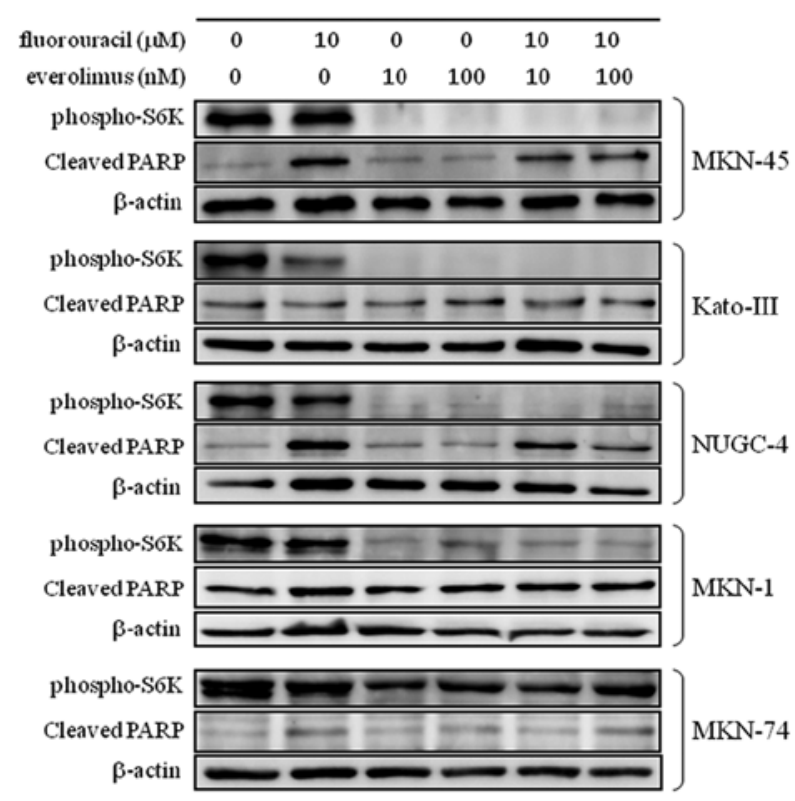

Figure 6. Expression of phosphorylated S6K and cleaved PARP in gastric cancer cell lines harboring genetic alteration in RTKs or signaling molecules other than HER2 with and without everolimus, fluorouracil, or their combination. Cells grown in $10 \%$ serum-containing media were lysed and immunoblotted for each protein. Blots were stripped and re-probed for $\beta$-actin as loading control.

In our study, we found that inhibition of the phosphorylation of S6K with treatment with trastuzumab occurred only in NCI-N87 cells, and not in MKN-7 cells. With regard to the phosphorylation of Akt, in contrast, trastuzumab induced no or only modest changes in both cell lines (Fig. 1B). This finding is consistent with our previous study using HER2amplified breast cancer cell lines, in which the degree of growth inhibition by trastuzumab was more closely correlated with inhibition of the phosphorylation of S6K than of $\mathrm{Akt}$ (30). These findings raise the possibility that phosphorylation of S6K might be a pharmacodynamic marker for anti-HER2 therapy in HER2-amplified cancer cells regardless of their origin.

We also found that a decrease in phosphorylation of S6K was necessary to enhance fluorouracil-induced apoptosis in HER2-amplified gastric cancer cell lines. While the mTORS6K signal has been characterized as involved in G1/S cell cycle progression by initiating protein translation, this signal is also known to promote cell survival. This latter effect was reported to occur via active S6K phosphorylation of the pro-apoptotic factor BAD at Ser136 (31). The primary role of S6K might differ depending on cell type. As we did not find that everolimus enhanced fluorouracil-induced apoptosis in gastric cancer cell lines which have genetic alteration in RTKs or signaling molecules other than HER2 (Fig. 6), a predominant role of $\mathrm{S} 6 \mathrm{~K}$ in cell survival might be characteristic in HER2-amplified gastric cancer cells. In some cell lines, the addition of everolimus rather decreased the level of fluorouracil-induced apoptosis compared to fluorouracil alone (Fig. 6). This might be due to the known effect of everolimus to induce cell cycle arrest, under which fluorouracil would not work effectively. Determining the predominant role of S6K in cell cycle progression vs. cell survival in specific cells might therefore be therapeutically important.

The clinical usefulness of everolimus is currently under investigation. A phase II clinical trial of everolimus monotherapy in patients with advanced gastric cancer showed a disease control rate of $56 \%$ despite no objective tumor response (32). Further, a randomized, double-blind, multi-center phase III study comparing everolimus plus best supportive care (BSC) with placebo plus BSC in patients with advanced gastric cancer after progression on 1 or 2 prior systemic chemotherapies is now ongoing (33). The results of this trial, particularly the subset analysis based on HER2 status, should provide deeper insight into the importance of the mTOR-S6K signal in the survival of HER2-amplified gastric cancers. Although the combination of capecitabine and everolimus showed only a modest effect in a phase I study (34), evaluating the trastuzumab-fluorouracil (or its derivative) combination after enrichment of the study population with patients whose tumors have HER2-amplification may be worthwhile.

Several limitations of this study warrant mention. First, our use of only two HER2-amplified gastric cancer cell lines precludes the generalization of the results. Compared to breast cancer, substantially fewer HER2-amplified gastric cancer cell lines are available. Confirmation of our findings in other or newly established HER2-amplified gastric cancer cell lines would be valuable.

Second, in addition to its inhibition of HER2 signaling, several studies have indicated the contribution of antigendependent cellular cytotoxicity (ADCC) in the antitumor effect of trastuzumab in breast cancer. Because ADCC only works in in vivo conditions, our present data do not necessarily deny the potential effect of trastuzumab on tumors showing resistance to trastuzumab in vitro.

In summary, our findings suggest that inhibition of the mTOR-S6K signal is a key molecular event in enhancing fluorouracil-induced apoptosis in HER2-amplified gastric cancer cells, regardless of sensitivity to trastuzumab. mTOR inhibitors such as everolimus might be attractive back-up drugs in a particular subset of gastric cancers. A better understanding of these findings, however, may require further investigation in clinical trials and associated translational studies.

\section{Acknowledgements}

This study was supported by the Global Centers of Excellence Program (H.M.) and Grant-in-Aid for Young Scientists (B) from the Ministry of Education, Culture, Sports, Science and Technology of Japan (T.M), and AstraZeneca Research Grant (T.M).

\section{References}

1. Jemal A, Bray F, Center MM, Ferlay J, Ward E and Forman D: Global cancer statistics. CA Cancer J Clin 61: 69-90, 2011.

2. Kamangar F, Dores GM and Anderson WF: Patterns of cancer incidence, mortality, and prevalence across five continents: defining priorities to reduce cancer disparities in different geographic regions of the world. J Clin Oncol 24: 2137-2150, 2006.

3. Sue-Ling HM, Johnston D, Martin IG, et al: Gastric cancer: a curable disease in Britain. BMJ 307: 591-596, 1993. 
4. Menges $M$ and Hoehler T: Current strategies in systemic treatment of gastric cancer and cancer of the gastroesophageal junction. J Cancer Res Clin Oncol 135: 29-38, 2009.

5. Ajani JA: Evolving chemotherapy for advanced gastric cancer. Oncologist 10 (Suppl 3): 49-58, 2005.

6. Slamon DJ, Leyland-Jones B, Shak S, et al: Use of chemotherapy plus a monoclonal antibody against HER2 for metastatic breast cancer that overexpresses HER2. N Engl J Med 344: 783-792, 2001.

7. Paez JG, Janne PA, Lee JC, et al: EGFR mutations in lung cancer: correlation with clinical response to gefitinib therapy. Science 304: 1497-1500, 2004.

8. Lynch TJ, Bell DW, Sordella R, et al: Activating mutations in the epidermal growth factor receptor underlying responsiveness of non-small-cell lung cancer to gefitinib. N Engl J Med 350: 2129-2139, 2004.

9. Yokota J, Yamamoto T, Miyajima N, et al: Genetic alterations of the c-erbB-2 oncogene occur frequently in tubular adenocarcinoma of the stomach and are often accompanied by amplification of the v-erbA homologue. Oncogene 2: 283-287, 1988.

10. Hofmann M, Stoss O, Shi D, et al: Assessment of a HER2 scoring system for gastric cancer: results from a validation study. Histopathology 52: 797-805, 2008.

11. Tanner M, Hollmen M, Junttila TT, et al: Amplification of HER-2 in gastric carcinoma: association with Topoisomerase II $\alpha$ gene amplification, intestinal type, poor prognosis and sensitivity to trastuzumab. Ann Oncol 16: 273-278, 2005.

12. Kuniyasu H, Yasui W, Kitadai Y, Yokozaki H, Ito H and Tahara E: Frequent amplification of the c-met gene in scirrhous type stomach cancer. Biochem Biophys Res Commun 189: 227-232, 1992.

13. Hattori $\mathrm{Y}$, Itoh $\mathrm{H}$, Uchino $\mathrm{S}$, et al: Immunohistochemical detection of K-sam protein in stomach cancer. Clin Cancer Res 2 1373-1381, 1996

14. Ueda T, Sasaki H, Kuwahara Y, et al: Deletion of the carboxylterminal exons of K-sam/FGFR2 by short homology-mediated recombination, generating preferential expression of specific messenger RNAs. Cancer Res 59: 6080-6086, 1999.

15. Tsujimoto H, Sugihara H,Hagiwara A and Hattori T: Amplification of growth factor receptor genes and DNA ploidy pattern in the progression of gastric cancer. Virchows Arch 431: 383-389, 1997.

16. Bang YJ, Van Cutsem E, Feyereislova A, et al: Trastuzumab in combination with chemotherapy versus chemotherapy alone for treatment of HER2-positive advanced gastric or gastro-oesophageal junction cancer (ToGA): a phase 3, open-label, randomised controlled trial. Lancet 376: 687-697, 2010.

17. Neve RM, Lane HA and Hynes NE: The role of overexpressed HER2 in transformation. Ann Oncol 12 (Suppl 1): 9-13, 2001.

18. Yokoyama H, Ikehara Y, Kodera Y, et al: Molecular basis for sensitivity and acquired resistance to gefitinib in HER2overexpressing human gastric cancer cell lines derived from liver metastasis. Br J Cancer 95: 1504-1513, 2006.

19. Matsui Y, Inomata M, Tojigamori M, Sonoda K, Shiraishi N and Kitano S: Suppression of tumor growth in human gastric cancer with HER2 overexpression by an anti-HER 2 antibody in a murine model. Int J Oncol 27: 681-685, 2005.
20. Lane HA, Beuvink I, Motoyama AB, Daly JM, Neve RM and Hynes NE: ErbB2 potentiates breast tumor proliferation through modulation of p27(Kip1)-Cdk2 complex formation: receptor overexpression does not determine growth dependency. Mol Cell Biol 20: 3210-3223, 2000.

21. Abramoff MD, Magalhaes PJ and Ram SJ: Image processing with ImageJ. Biophotonics Int 11: 36-42, 2004.

22. Pegram MD, Konecny GE, O'Callaghan C, Beryt M, Pietras R and Slamon DJ: Rational combinations of trastuzumab with chemotherapeutic drugs used in the treatment of breast cancer. J Natl Cancer Inst 96: 739-749, 2004.

23. Chou TC and Talalay P: Quantitative analysis of dose-effect relationships: the combined effects of multiple drugs or enzyme inhibitors. Adv Enzyme Regul 22: 27-55, 1984.

24. Gong SJ, Jin CJ, Rha SY and Chung HC: Growth inhibitory effects of trastuzumab and chemotherapeutic drugs in gastric cancer cell lines. Cancer Lett 214: 215-224, 2004.

25. Yokozaki H: Molecular characteristics of eight gastric cancer cell lines established in Japan. Pathol Int 50: 767-777, 2000.

26. Mita H, Toyota M, Aoki F, et al: A novel method, digital genome scanning detects KRAS gene amplification in gastric cancers: involvement of overexpressed wild-type KRAS in downstream signaling and cancer cell growth. BMC Cancer 9: 198, 2009.

27. Sharma P and Halayko AJ: Emerging molecular targets for the treatment of asthma. Indian J Biochem Biophys 46: 447-460, 2009.

28. Kim SY, Kim HP, Kim YJ, et al: Trastuzumab inhibits the growth of human gastric cancer cell lines with HER2 amplification synergistically with cisplatin. Int J Oncol 32: 89-95, 2008.

29. Tanizaki J, Okamoto I, Takezawa K, et al: Synergistic antitumor effect of S-1 and HER2-targeting agents in gastric cancer with HER2 amplification. Mol Cancer Ther 9: 1198-1207, 2010.

30. Kataoka Y, Mukohara T, Shimada H, Saijo N, Hirai M and Minami H: Association between gain-of-function mutations in PIK3CA and resistance to HER2-targeted agents in HER2amplified breast cancer cell lines. Ann Oncol 21: 255-262, 2010.

31. Harada H, Andersen JS, Mann M, Terada N and Korsmeyer SJ: p70S6 kinase signals cell survival as well as growth, inactivating the pro-apoptotic molecule BAD. Proc Natl Acad Sci USA 98: 9666-9670, 2001.

32. Doi T, Muro K, Boku N, et al: Multicenter phase II study of everolimus in patients with previously treated metastatic gastric cancer. J Clin Oncol 28: 1904-1910, 2010.

33. Danilkovitch-Miagkova A and Zbar B: Dysregulation of Met receptor tyrosine kinase activity in invasive tumors. J Clin Invest 109: 863-867, 2002.

34. Lim T, Lee J, Lee DJ, et al: Phase I trial of capecitabine plus everolimus (RAD001) in patients with previously treated metastatic gastric cancer. Cancer Chemother Pharmacol 68: 255-262, 2011. 